\title{
En búsqueda del pasado y futuro del laicado Proyecciones latinas a partir del testimonio de Marcos McGrath y Lucio Gera
}

\author{
The Past and Future of the Laity: \\ Thinking latinamente about the Witnesses \\ of Marcos McGrath and Lucio Gera
}

Peter Casarella

\section{Resumen}

Los teólogos latinos en los EE.UU. han desarrollado un programa para la eclesiología, pero dentro de esta literatura poco se ha sistematizado sobre la teología del laicado. Este ensayo inicia un diálogo con la teología latinoamericana del período del Concilio Vaticano Segundo y sus momentos anteriores e inmediatamente posteriores. ElArzobispo Marcos McGrath, C.S.C. introdujo un nuevo método para el análisis de los signos de los tiempos (véase Gaudium et Spes, N.4) y, especialmente, presentó la necesidad de promover la acción de los pobres y otros laicos marginados y de predicar su liberación de la opresión económica. La figura de Lucio Gera introdujo el concepto del pueblo y una teología basada en la fe y en la experiencia del pueblo de Dios. Ambos teólogos han contribuido de forma original al desarrollo de una teología del laicado en el contexto latinoamericano. Se sostiene que estas dos aproximaciones a la promoción del laicado son fundamentales para el futuro de la eclesiología latina en los EE.UU, y que lo/as hispano/as laico/as serán 
los beneficiarios de los dos modelos latinoamericanos y de su convergencia en la experiencia hispana.

Palabras claves: Laicado. Lucio Gera. Marcos McGrath. Gaudium et Spes. Signos de los tiempos.

\begin{abstract}
Latino/a theologians have only begun to develop a program for ecclesiology, and within that literature virtually nothing is said about theology of the laity. This essay undertakes a dialogue with Latin American theology from the period just before, during, and just after the Second Vatican Council. The figure of Archbishop Marcos McGrath, C.S.C. introduces the new method for analyzing the signs of the times (cf. Gaudium et Spes, \#4) and especially the need to promote the agency of poor and marginalized lay persons and their liberation from economic oppression. The figure of Lucio Gera introduces the concept of "the people" and a theology based upon the faith and experience of the people of God. Both figures contributed seminally to the development of a theology of the laity in a Latin American context. It is argued here that both approaches to promoting the laity are critical for the future of Latino/a ecclesiology and that Hispanic lay persons will benefit from thinking about the two approaches from Latin America and their convergence in the experience of Latino/as.
\end{abstract}

Keywords: Laity. Lucio Gera. Marcos McGrath. Gaudium et Spes. Signs of the times.

\title{
1. Preguntas abiertas en la eclesiología de los latino/as en los EE.UU.
}

Los católicos en los EE.UU. ya reconocen que su futuro es mestizo. ${ }^{1}$ Los hispanos están presentes prácticamente en todas las diócesis en Estados Unidos, y el porcentaje de los hispanos en la iglesia católica estadounidense es más de un $35 \%{ }^{2}$ Según numerosas proyecciones, este número aumentará

\footnotetext{
${ }^{1}$ Véase ELIZONDO, Virgilio. El futuro es mestizo: vivir donde se juntan las culturas. San Antonio: Centro Cultura Mexicano Americano, 2000.

${ }^{2}$ Este porcentaje sale en el sitio de la conferencia episcopal estadounidense pero es basado en un estudio demográfico del Centro Pew del abril de 2010. Disponible en: $<$ http://www.usccb.
} 
en el futuro. ${ }^{3}$ Este crecimiento tendrá lugar a pesar de la salida notable de los católicos de la iglesia, un acontecimiento nuevo que fue reportado recientemente por el Centro de Investigación de Pew. ${ }^{4}$ Todos estos cambios crean una situación muy fluida en la que la iglesia católica estadounidense se hace a la vez más hispana y más secular. El aporte de los hispanos no puede ser ignorado, pues serán ellos, que ya representan una población bastante joven, quienes probablemente conformen en la próxima generación la mitad de la iglesia nacional.

¿Hay un pensamiento eclesial que le corresponda a este enorme cambio demográfico? Un punto de partida para reflexionar sobre los laicos hispanos son los encuentros nacionales. Hace poco fueron publicados dos trabajos sobre los Encuentros Nacionales Hispanos de los años 70 y 80, que muestran la actualidad e importancia de la contribución hispana a la pastoral. El evento enfatizó a los hispanos como agentes pastorales, y no sólo como recipientes pasivos de la pastoral. En los encuentros los líderes hispanos utilizaron el método de teología en conjunto, que llegó a ser una característica clave de la teología latina. Durante los encuentros, grupos pequeños discutieron la situación de los hispanos y después sintetizaron los resultados de las llamadas listening sessions en resoluciones plenarias. ${ }^{5}$

org/issues-and-action/cultural-diversity/hispanic-latino/demographics/datos-demograficossobre-los-catolicos-hispanos-latinos-en-estados-unidos.cfm>.

${ }^{3}$ Véase MATOVINA, Timothy. Latino Catholicism: Transformation in America's Largest Church. Princeton: Princeton University Press, 2012.

${ }^{4}$ Véanse por ejemplo los estudios descritos en estos sitios. Disponible en: $<$ http://www.catholic. org/news/national/story.php?id=60423 y http://www.pewresearch.org/fact-tank/2014/11/24/ different-destinations-for-u-s-hispanics-latin-americans-who-leave-catholic-church/>. $\quad$ En resumen, los estudios sociológicos muestran que en 2007 eran 54 millón católicos en los EE.UU. En 2015 se quedan 51 millón. Un porcentaje creciendo de los ex-católicos escogen "nada" como su preferencia religiosa y por eso son llamados los "nones". El rechazo de toda religión institucional representa un relativamente nuevo fenómeno. A pesar de la salida de muchos católicos de la iglesia, el número de los latinos hispanos católicos sigue creciendo a causa principalmente de las inmigraciones de América Latina a los EE.UU. Disponible en: $<$ http://www.pewresearch.org/fact-tank/2014/05/07/fewer-hispanics-are-catholic-so-how-canmore-catholics-be-hispanic/>.

${ }^{5}$ La historia y eclesiología de los Encuentros Nacionales es un tema que sólo ahora se elabora en estudios académicos. Véase, por ejemplo, TAMPE, Luis A., S.J. Encuentro Nacional Hispano de Pastoral (1972-1985): An Historical and Ecclesiological Analysis, Ph.D. dissertation, The Catholic University of America, 2014 y Mario J. Paredes, The History of the National Encuentros: Hispanic Americans in the One Catholic Church. Mahwah, N.J.: Paulist Press, 2015. 
El primer encuentro tuvo lugar en 1972 y logró promover una mayor participación de los católicos hispanos en la vida y misión de la Iglesia, incluyéndolos en posiciones de liderazgo y en los procesos de toma de decisiones. También se establecieron estructuras específicas para servir a los hispanos. En el Segundo Encuentro Nacional Hispano se formularon recomendaciones para una Iglesia más sensible, multicultural, espiritualmente viva, unida y creativa; a través de un proceso de evangelización. En 1983 los obispos estadounidenses publicaron una carta pastoral sobre "La Presencia Hispana: Esperanza y Compromiso". En la carta hacen un llamado a los católicos hispanos a levantar de nuevo sus voces proféticas. En el Tercer Encuentro Nacional Hispano, celebrado en 1985, participaron más de medio millón de hispanos en una consulta popular que condujo a la elaboración del Plan Pastoral Nacional para el Ministerio Hispano; plan que en 1987 fue aprobado por unanimidad por los obispos católicos. El Plan contempla las prioridades y acciones para el ministerio hispano a nivel parroquial, diocesano y regional.

Los Encuentros Nacionales son un buen modelo para reflexionar sobre las tareas de una nueva eclesiología. Sin embargo, trabajos por los teólogos hispanos sobre la eclesiología son pocos. En 1995, Orlando Espín escribió un ensayo sobre ciertas semejanzas entre el Pentecostalismo y el catolicismo popular ${ }^{6}$. En 1999, Gary Riebe-Estrella aclaró el concepto de pueblo de Dios en la vida cotidiana de los latinos ${ }^{7}$. Alejandro García-Rivera escribió reflexiones importantes en su libro de 1999, The Community of the Beautiful, y también en otro ensayo sobre un nueva nota de la iglesia ${ }^{8}$. Finalmente, Roberto Goizueta contribuyó en 2007 con un ensayo clave: "Corpus verum: Hacia una eclesiología fronteriza". ${ }^{9}$ En este artículo Goizueta aborda la crítica de Jon Sobrino, según la cual el concepto del pueblo de Dios relativiza las grandes

\footnotetext{
${ }^{6}$ ESPÍN, Orlando. "Pentecostalism and Popular Catholicism: The Poor and Traditio" Journal of Hispanic/Latino Theology 3, no. 2 (1995), pp. 14-43.

${ }^{7}$ RIEBE-ESTRELLA, Gary. "Pueblo and Church". In: ESPÍN, Orlando O.; DÍAZ, Miguel H. (eds.). From the Heart of Our People: Latino/a Explorations in Catholic Systematic Theology. Maryknoll, NY: Orbis Books, 1999, pp. 172-88.

${ }^{8}$ GARCÍA-RIVERA, Alejandro. The Community of the Beautiful: A Theological Aesthetics. Collegeville, MN: Liturgical Press, 1999, y GARCÍA-RIVERA, Alejandro. "The Church is Beautiful and Holy". In: MADGES, William; DALEY, Michael J. (eds.). The Many Marks of the Church. New London: Twenty-Third Publications, 2006, pp. 69-73.

9 GOIZUETA, Roberto. "Corpus Verum: Toward a Borderland Ecclesiology". Journal of Hispanic/Latino Theology (December 2007). Disponible en: <http://www.latinotheology.org/ node/32/print $>$.
} 
desigualdades que se encuentran entre los laicos. La teología latina del laicado tiene que ser pensada más allá que una mera oposición entre la iglesia de los pobres y el pueblo de Dios. Goizueta enfatiza además que la estructura de liderazgo entre los Hispanos no es masculina y clerical, sino femenina y laical. Las estructuras de educación y catequesis deben ser repensadas sin apoyar el clericalismo y sin introducirlo escondidamente en la forma de pensamiento que inspira a los laicos.

De allí se evidencian características destacadas de la iglesia latina en los EE.UU.:

$>$ La práctica de la fe del pueblo todavía muestra una herencia medieval que deriva de la religiosidad popular pretridentina y premoderna. En cambio, las devociones populares en los EE.UU. derivadas de las nacionalidades europeas no se plantea actualmente como una dimensión pública del catolicismo.

$>$ La mentalidad del pueblo es, sin embargo, muy progresiva y por eso no hay ninguna tentación al revanchismo entre la mayoría de los latinos.

$>$ El sentido de la fe (sensus fidei) es opuesto a la religión del clero y todas las estructuras neocoloniales. El liderazgo del pueblo como pueblo viene principalmente del laicado y de las mujeres.

$>$ La población hispana sigue siendo una iglesia de los pobres y marginados, en las palabras de Roberto Goizueta, una ecclesia crucis.

$>$ Sobre todo, la fe latina es opuesta al catolicismo puritano de los EE.UU. En la práctica de la fe, no hay una separación rígida entre la doctrina y símbolo. La fe latina presupone lo que Goizueta denomina "un realismo simbólico". ${ }^{10}$

Los estudios no abordan todas las cuestiones de la eclesiología ni en el sentido de los tratados más tradicionales ni en torno a la experiencia actual del pueblo. Por eso, quedan unas preguntas abiertas:

$>$ El pueblo de Dios latino no representa en ninguna manera un

${ }^{10}$ GOIZUETA, Roberto S. "The Symbolic Realism of U.S. Popular Catholicism". Theological Studies 65 (2004), pp. 255-74. 
laicismo anticlerical. Entonces, ¿cómo se promueve la colaboración entre el clero y los laicos?

$>$ La teología del pueblo fortalece el pueblo de Dios como un concepto y como una realidad social, pero ¿qué quiere decir ser un pueblo en el contexto norteamericano?

$>$ Hay un nuevo protagonismo del pueblo santo, fiel a Dios en la historia actual. Pero, ¿cómo se interpreta su papel frente a los signos de los tiempos actuales?

$>$ ¿Cómo mantiene el pueblo un equilibrio entre su religiosidad premoderna y -por lo menos en parte- indígena, y su lucha progresista y muy moderna contra la injusticia y exclusión social? ¿No parece ser una paradoja total ante los ojos de los ciudadanos estadounidenses?

$>$ ¿Cuál es entonces la tarea principal de los latinos/as, luchar para la liberación social o promover la nueva evangelización utilizando los recursos de la teología del pueblo?

\section{El descubrimiento desde América Latina de una teología de los signos de los tiempos}

La teología latina es el fruto de una nueva forma de pensamiento. Refleja los signos de los tiempos. La reflexión teológica latina no termina con los estudios de investigadores aislados sino que crece con y desde el pueblo latino de Dios. Es una forma de pensar "latinamente". ${ }^{11}$ Por eso, es muy sugerente y fructífero el comparar la tarea de teologizar latinamente con el desafío conciliar que se encuentra en la Constitución Pastoral de la Iglesia, a lo cual muchos teólogos latinoamericanos se han dedicado; o sea, la lectura de los signos de los tiempos y la interpretación de aquellos signos a la luz del Evangelio. ${ }^{12}$

Muchas características de la lectura latinoamericana de los signos de los tiempos resuenan en la teología latina. La génesis del pensamiento sobre los signos de los tiempos en el contexto latinoamericano comienza con la figura

\footnotetext{
${ }^{11}$ La formulación "doing theology latinamente" se encuentra con frecuencia en las obras de los teólogos latinos.

${ }^{12}$ Véase Gaudium et spes, \#4.
} 
de Marco McGrath. ${ }^{13}$ Marcos McGrath nació en 1924 en la Zona del Canal de Panamá. ${ }^{14}$ Su padre era estadounidense y fue a Panamá para trabajar en el Canal, donde contrajo matrimonio con Louise Renauld, quien llegó junto con su hermano desde su hogar en Costa Rica.

Marcos estudió en la Universidad de Notre Dame entre 1940 y 1942, tiempo en que, según Robert Pelton, fue introducido a la Acción Católica y al papel importante del laico dentro de la iglesia. ${ }^{15}$ A fines del verano de 1942, McGrath ingresó al Noviciado de la Congregación de la Santa Cruz. En 1953 terminó su tesis de doctorado en el Angelicum de Roma sobre la doctrina del Concilio Vaticano en la evolución del dogma. ${ }^{16}$ Esta obra de su juventud es muy erudita y bastante iluminadora respecto de la participación futura de McGrath en el Vaticano II. Muestra el equilibrio que mantuvo el Concilio Vaticano I con el nuevo liberalismo del siglo XIX, a saber, Georg Hermes (1775-1835) y tendencias conservadoras como el fideísmo de Louis Eugène Marie Bautain (1796-1867) y Felicité de Lammennais (1782-1854). ${ }^{17}$ Un comentario breve hecho por McGrath en el prólogo revela que su propia orientación viene de los modelos orgánicos del desarrollo de la doctrina del siglo XIX, es decir, del pensamiento de John Henry Newman y Johann Adam Möhler. ${ }^{18}$ El contenido de la tesis es sobre las declaraciones del Concilio Vaticano I y la situación de la teología católica entre 1800 y 1831, pero la metodología proviene de la relectura de Newman y Möhler, realizada por teólogos del siglo veinte que McGrath ha citado ampliamente, como por ejemplo, Yves Congar

\footnotetext{
${ }^{13}$ SCHICKENDANTZ, Carlos. "Una elipse con dos focos. Hacia un nuevo método teológico a partir de Gaudium et Spes”. In: AZCUY, Virginia Raquel; SCHICKENDANTZ, Carlos; SILVA, Eduardo (eds.). Teología de los Signos de los Tiempos Latinoamericanos. Horizontes, Criterios, y Métodos. Santiago de Chile: Ediciones Universidad Alberto Hurtado, 2013, pp. 53-87.

${ }^{14}$ PELTON, Robert S., C.S.C. El obispo Marcos McGrath, C.S.C. Santiago de Chile: Talleres de E.P., 1966, p. 5.

15 Ibid., 6.

${ }^{16}$ MCGRATH, Marcos G., C.S.C. The Vatican Council's Teaching on the Evolution of Dogma: A Study in Nineteenth Century Theology. Rome: Pontificum Athenaeum Angelicum, 1953.

${ }^{17}$ Ibid., 56-8. McGrath nota la diferencia entre Lammennais y Newman en p. 62, n. 75-76.

${ }^{18}$ MCGRATH, Marcos G., C.S.C. The Vatican Council's Teaching on the Evolution of Dogma, 11. Möhler escribió Symbolik en 1833, y Newman publicó An Essay on the Development of Christian Doctrine en 1846. McGrath menciona que según Newman de Maistre y Möhler son los precursores de un modelo orgánico del desarrollo de la doctrina. M. McGrath, The Vatican Council's Teaching, 18, citando NEWMAN, J.H. An Essay on the Development of Christian Doctrine. London, 1927, p. 97.
} 
O.P. ${ }^{19}$. La tesis refleja las semillas de la confianza impresionante que mostró McGrath frente a los nuevos desafíos a partir de la revolución social de 1968, y la necesidad de promover progreso orgánico en la historia de iglesia durante y después del Concilio Vaticano II. McGrath, entonces, aprendió los principios de su teoría del progreso moderno a partir de un estudio crítico del Concilio Vaticano I y su contexto teológico.

En abril del mismo año que terminó su tesis, McGrath fue enviado por su Congregación a trabajar en el colegio Saint George, en Santiago de Chile. ${ }^{20}$ Fundó las obras sociales de Saint George en 1954. Según Pelton, la presencia de dos clases sociales totalmente opuestas entre sí, una al lado de la otra significó una sorpresa total para McGrath. Pelton señala: "[1]os primeros encuentros de los miembros de las Obras Sociales San Jorge con los pobres produjeron experiencias desgarradoras, amistades y los comienzos de ayuda material." ${ }^{21}$ Hay varios elementos destacados e importantes que ya emergen de esta pastoral: la dirección espiritual de los jóvenes con catequesis y lecturas bíblicas, la opción preferencial por los pobres y junto con ésta, el reconocimiento de la necesidad de iniciar la pastoral con fraternidad personal; y el empoderamiento de los laicos para que puedan asumir su propio papel en la iglesia.

En 1954, McGrath comenzó a enseñar en la Facultad de Teología de la Universidad Católica de Chile. En 1959 fue nombrado Decano de dicha facultad y en 1960 fundó la Revista Teología y Vida. Su colaboración en estos años con el obispo chileno Manuel Larraín en el campo de justicia social fue notable. ${ }^{22}$ Aunque fue un ciudadano estadounidense que estudió teología en los Estados Unidos, Roma y Paris, su compromiso teológico de esta época refleja una teología no culturada, muy a favor de la perspectiva chilena:

La palabra "teología" suscita en muchos el espectro medieval de extrañas

\footnotetext{
${ }^{19}$ En un ensayo publicado en 1960, McGrath denominó a "Journet, Congar, Rahner, GarrigouLaGrange, y otros" como "nuestros grandes teólogos de hoy”. Marcos McGrath, “¿Qué es la teología?" Teología y Vida 1 (1960), p. 5. Tres de los cuatro son dominicos franceses, un hecho que refleja su tiempo de estudio en el Institut Catholique de Paris y en el Angelicum.

${ }^{20}$ PELTON, Robert S. El obispo Marcos McGrath, p. 16.

${ }^{21}$ Ibid., p. 17.

${ }^{22}$ PELTON, Robert. El obispo Marcos McGrath, 20-21. En Chile el obispo de Talca Larraín y el jesuita Padre Alberto Hurtado eran forjadores del catolicismo social antes del Concilio. Véase BERRÍOS, Fernando. “Antecedentes y recepción de Gaudium et Spes en Latinoamérica. Una mirada desde Chile”. In: Teología de los Signos de los Tiempos Latinoamericanos, pp. 28-33.
} 
teorías sobre doctrinas y opiniones de poca o ninguna relación al hombre y su labor diaria. Si pretendemos, pues, relacionar en un mismo título de revista estos dos términos al parecer antagónicos, preciso es que dediquemos este primer número en gran parte a mostrar que, para quienes profesan creer en Dios, no pueden oponerse... Para vivir como hombre es imprescindible pensar. Para vivir como cristiano es indispensable pensar la Fe. Para nosotros no basta que la Fe se esté pensando en Roma o en París o en Munich. Es preciso que la pensemos en Chile. ${ }^{23}$

El nombre de la revista era entonces un programa preconciliar para repensar la relación entre la fe, lo cotidiano y la inculturación. La revista continúa hoy con mucha vitalidad, pese a que McGrath no pudo participar en el proyecto chileno de vincular fe y vida por mucho tiempo. McGrath tuvo que salir de Chile en 1961 porque fue nombrado obispo auxiliar en Panamá y pocos años después tuvo que participar en el Concilio Vaticano II.

En 1964 participó en una reunión en Chicago, en la que tuvo que hablar frente a seis cardenales y otros miembros de la jerarquía, además de muchas personas religiosas y seculares de Norte y Sudamérica. Tocó el tema de los laicos. Sus palabras mantienen su actualidad hoy porque la situación desafortunadamente no ha cambiado mucho:

Nos encontramos actualmente a la vanguardia de una gran ola de acción cooperativa interamericana...Este rol de la Iglesia Católica en nuestro hemisferio es una verdad evidente que muchos entusiastas del acercamiento interamericano parecen querer olvidar. ${ }^{24}$

El papel de la iglesia norteamericana en los años 60 comprendió el enviar misioneros del norte al sur. Sin embargo, el enfoque de McGrath no estaba orientado al modelo unilateral de desarrollo, ni de la iglesia ni de la economía. $\mathrm{Su}$ enfoque principal era la educación interamericana con el apoyo fuerte de los pueblos de ambos lados. Producto de la pura casualidad del sitio de su nacimiento y de su herencia bicultural, McGrath tuvo el don de una educación interamericana que lo hizo querer promover un mejor nivel de intercambio educacional para toda la iglesia en América. ${ }^{25}$

\footnotetext{
${ }^{23}$ MCGRATH, Marcos. "Editor fundante”. Teología y Vida Año 1, № 1 (1960), p. 4.

${ }^{24}$ Pelton, Robert. El obispo Marcos McGrath, 9-10.

${ }^{25}$ Repito aquí una expresión famosa y todavía desafiante de una exhortación apostólica de San Juan Pablo. Sobre la Ecclesia en América como principio ecclesial, véase por ejemplo:
} 
McGrath participó en la Comisión Doctrinal del Concilio Vaticano II y tenía un conocimiento muy íntimo de todas las etapas de la elaboración de la Constitución Pastoral Gaudium et Spes. Al comienzo de la primera sesión, McGrath fue elegido para un cargo en la Comisión Teológica, siendo uno de los tres prelados latinoamericanos elegidos para este fin. ${ }^{26}$ Después del Concilio se dedicó a la aplicación de la nueva pastoral del Concilio a la realidad interamericana y, sobre todo, latinoamericana. ${ }^{27} \mathrm{En}$ su lectura del evento del Concilio, McGrath enfatizó el estilo único de Gaudium et Spes. ${ }^{28}$ "Nunca antes un Concilio había abordado los aspectos directamente temporales de la vida cristiana de una manera tan extensa y sistemática", dijo McGrath. ${ }^{29}$ En el conflicto que tuvo lugar entre los que querían un tratamiento puramente doctrinal y los que querían enfrentarse con los signos de los tiempos, McGrath defendió “el nuevo método para este nuevo tipo de documento." ${ }^{30}$ Aunque el documento no refiere directamente a los métodos de las nuevas ciencias, McGrath evidencia que en la nota 62 del documento "está lleno de referencias al acercamiento de la teología con las diversas manifestaciones de la ciencia y de la cultura de hoy: un acercamiento que se presenta como necesario y ventajoso para ambos". ${ }^{31}$ El concepto de diálogo introducido por Pablo VI en Ecclesiam Suam es mencionado no solamente para apoyar el ecumenismo entre católicos y otros cristianos, sino también para mostrar el nuevo desafío metodológico

CASARELLA, Peter. "Solidarity as the Fruit of Communion: Ecclesia in America, 'PostLiberation Theology,' and the Earth”. Communio: International Catholic Review 27 (2000), pp. 98-123.

26 PELTON, Roberto. El obispo Marcos McGrath, 36. La participación de McGrath en el desarrollo de la teología de los signos de los tiempos durante el concilio es relatada en Giovanni Turbanti, "Commissione Mista per lo Schema XVII-XIII," en Les Commissions Conciliares à Vatican II, ed. M. Lamberigts, Cl. Soetens, y J. Grootaers. Leuven: Bibliotheek van de Faculteit Godgeleerdheid, 1996, p. 237. Turbanti confirma en n. 41 que el Cardenal Karol Wojtyła de Polonia era un miembro de la subcomisión presidida por McGrath.

27 Véase, por ejemplo, MCGRATH, Marcos. "Un nuevo método de pastoral de la Iglesia". Criterio 1543 (14 de marzo de 1968), pp. 134-37; MCGRATH, Marcos. Los Signos de los Tiempos en América Latina Hoy =el discurso de McGrath como Vice-Presidente de CELAM durante la Conferencia General en Medellín. Santiago de Chile: Oficina de Catequesis, 1968; y MCGRATH, Marcos. "La Génesis de 'Gaudium et Spes”". Mensaje 153 (1966), pp. 495-502.

28 "La Génesis", p. 495.

${ }^{29}$ Ibid., p. 496.

${ }^{30}$ Ibid.

${ }^{31}$ Ibid., p. 498. 
que abrió el Concilio. ${ }^{32}$ En suma, el nuevo método propone un "enfoque a la vez empírico y teológico." ${ }^{33}$

McGrath acuñó la expresión "nuevo método para este nuevo tipo de documento" y así abrió una nueva pista para América Latina y para la iglesia. Muchos teólogos de hoy son beneficiarios de esta apertura y reconocen además el aporte de McGrath en el cambio de paradigma. Carlos Schickendantz, un teólogo argentino que trabaja actualmente en el Instituto Teológico Manuel Larraín en Santiago de Chile, nos recuerda que la génesis durante el Concilio Vaticano II de la teología de los signos de los tiempos comienza con "el nuevo método"; articulado por primera vez y defendido por Marcos McGrath. ${ }^{34}$ En cierto modo, McGrath no era sólo un modelo de la construcción de vínculos entre el Norte y el Sur, sino también un testimonio forjador de una tarea aún más desafiante: la de sintetizar los dos modos de pensar. Schickendantz no es el único que recuerda esta herencia. Muchos latinoamericanos han reflexionado sobre la cuestión de la teología de los signos de los tiempos desde América Latina. Virginia R. Azcuy, por ejemplo, nombra la tarea principal del Instituto Teológico Manuel Larraín en Santiago de Chile como interpretatio temporis. ${ }^{35}$ Según ella, la lectura interpretativa de los signos conduce a "una topología plural", según la cual somos invitados a hacer nuestros propios "estudios locales." De ninguna manera, mantiene Azcuy, puede permitirse a una teología de los signos el alejarse del acontecimiento postconciliar que tuvo lugar en la Conferencia de Medellín, o sea, la autoconciencia y el llamado a ser una iglesia de los pobres. ${ }^{36}$ Fredy Parra explora por su parte el desafío temporal y existencialista como punto de partida de una teología latinoamericana de los signos de los tiempos ${ }^{37}$. Es muy relevante la sugerencia hecha por Parra

\footnotetext{
${ }^{32}$ Ibid., p. 502.
}

${ }^{33}$ Ibid., p. 501.

${ }^{34}$ SCHICKENDANTZ, Carlos. "Una elipse con dos focos", pp. 56-8.

${ }^{35}$ AZCUY, Virginia R. "La manifestación salvífica de Dios y su discernimiento en los signos de los tiempos. El giro soteriológico-pastoral en el Concilio Vaticano II". Teología y Vida 60 (2014), pp. 329-50, aquí citando p. 347.

${ }^{36}$ AZCUY, Virginia. "La Pobreza de la Iglesia y los signos de los tiempos. Medellín como recepción inacabada del Vaticano II". Teología de los Signos de los Tiempos Latinoamericanos, pp. 89-126.

${ }^{37}$ PARRA, Fredy. "Desafio del tiempo, y memoria, en Signos de Estos Tiempos", pp. 47-82 y más ampliamente en PARRA, Fredy. Esperanza en la Historia. Idea Cristiana del Tiempo. Santiago de Chile: Ediciones Universidad Alberto Hurtado, 2011, pp. 185-237. El planteamiento de Parra converge en cierto modo con el punto de partido del teólogo latino LEE, Michael. Véase por ejemplo su ensayo "Liberation Theology's Transcendent Moment: The Work of 
de iniciar de nuevo la lectura de la filosofía de Xavier Zubiri y la revolución zubiriana a favor de una filosofía de la posibilidad.

No es posible en este ensayo abordar todos los acercamientos a los signos de los tiempos en la reflexión latina. Un comentario breve sobre el punto de partida debe ser suficiente. Un signo muy importante es el mestizaje. El futuro de la presencia hispana depende en cierto modo en el reconocimiento del don de ser mestizo, una tarea que supone un desafío grande ${ }^{38}$. Elizondo aplica este reconocimiento cultural en El Caminar del Galileo (primera edición, 1983) a la cristología ${ }^{39}$, y así promueve la bendición del mestizaje del pueblo de Dios a través de una lectura hispana de la imagen Bíblica de la Galilea de los Gentiles. ${ }^{40}$ Esto se llama el "principio de Galilea", según Elizondo. En la última parte del libro define "el principio de la resurrección" como la esperanza del pueblo de celebrar escandalosas fiestas para iniciar la nueva vida de Jesucristo y del pueblo que él ha redimido del pecado y de la opresión.

El fundamento teologal más significativo para los latinos es entonces el Dios de sorpresas increíbles, un aporte que corresponde a la sugerencia de Parra de explorar la categoría filosófica de posibilidad. ${ }^{41}$ Así es como llegamos a la cuestión de la escatología cristiana. En realidad, la teología latina en su planteamiento escatológico oscila entre el proyecto histórico plenamente utópico de Ada María Isasí-Díaz, y la posición más realista de Alejandro

Xavier Zubiri and Ignacio Ellacuría as Non-Contrastive Discourse". Journal of Religion 83 (2003), pp. 226-43.

${ }^{38}$ ELIZONDO, Virgil. The Future is Mestizo: Life where Cultures Meet. Bloomington, IN: Meyer Stone Books, 1988. Este libro elabora in inglés lo que Elizondo publicó antes en L'avenir est au métissage (Paris: Nouvelle Editions Mame, 1987).

${ }^{39}$ The Galilean Journey: The Mexican-American Promise. Maryknoll, New York: Orbis, fue publicado en 1983. Algo viene de su tesis doctoral escrita en Paris bajo el título Métissage. Una segunda revisada edición del Galilean Journey fue publicado por Orbis en 2000. En 2002 Hermana Rosa María Icaza de MACC hizo la primera traducción en español: El Caminar del Galileo: La Promesa México-Americana. San Antonio: MACC, de la segunda edición.

${ }^{40}$ Isaías 9, 1-2 y Mateo 4, 15-16. Véase también la homilía que predicó Papa Francisco en la Basílica de San Pedro durante la Vigilia de Pascua de 2014: "En la vida del cristiano, después del bautismo, hay también una "Galilea" más existencial: la experiencia del encuentro personal con Jesucristo, que me ha llamado a seguirlo y participar en su misión...Hoy, en esta noche, cada uno de nosotros puede preguntarse: ¿Cuál es mi Galilea? ¿Dónde está mi Galilea?... "Galilea de los gentiles" (Mt 4,15; Is 8,23): horizonte del Resucitado, horizonte de la Iglesia; deseo intenso de encuentro... ¡Pongámonos en camino!”

${ }^{41}$ ELIZONDO, Virgilio. Jesús de Galilea: Un Dios de Sorpresas Increíbles. Chicago: Loyola University Press, 2007. 
García-Rivera. ${ }^{42}$ La escatología no debe enfocarse solamente en un tiempo del futuro. También hay una escatología de lo cotidiano en el presente. La fenomenología como aporte metodológico a una teología de los signos de los tiempos propuesta por José Daniel López, S.J., al menos hasta ahora, no se corresponde con los métodos explícitos de la teología latina; aunque la reflexión teológica latina, a partir de la experiencia en lo cotidiano y el testimonio de la religiosidad popular urbana, sugiere algo semejante. ${ }^{43}$

\section{Lucio Gera y el nacimiento de la teología del pueblo.}

Un desarrollo simultáneo con el desarrollo de la teología de los signos de los tiempos fue la emergencia en Argentina de la llamada teología del pueblo. En realidad, no es una novedad en sí, pues deriva de una categoría Bíblica que se encuentra en muchos sitios, incluso en Lumen Gentium, capítulo 2. Pero la articulación de la teología del pueblo por el Padre Lucio Gera avanzó la causa de los laicos y será por eso el enfoque de esta sección.

Por medio de la promoción de una cultura de encuentro, el teólogo del pueblo escucha las voces del pueblo santo, fiel a Dios, y sobre todo, las voces de lo/as laico/as. En torno al método teológico de la teología del pueblo, hay tres ejes importantes. Primero, promover la reflexión sobre las necesidades prácticas y pastorales del pueblo de Dios, prestando atención especial al grito de los pobres en la ciudad. Segundo, discernir la sabiduría teológica inscrita en la religiosidad popular en toda su variedad con un enfoque dialógico y purificador en el encuentro con la persona de Jesucristo. Tercero, formular una teología de cultura arraigada en los distintos sitios y objetos de devoción y creatividad donde se encuentra la fe del pueblo.

Lucio Gera es considerado el forjador principal de la teología del pueblo en Argentina. Su papel único es reconocido no sólo en su país, sino también a través de su compromiso teológico dentro de CELAM. Oscar Rodríguez

\footnotetext{
${ }^{42}$ Un ejemplo del pensamiento útopico de Isasí-Diaz es su ensayo ISASÍ-DÍAZ, Ada María. "La Habana: The City that Inhabits Me". In: Spirit in the Cities. ed. Kathryn Tanner. Minneapolis: Augsburg, 2004. El metódo realista de García-Rivera se encuentra en su libro The Garden of God: A Theological Cosmology. Minneapolis: Fortress Press, 2009.

${ }^{43}$ LÓPEZ, José Daniel. “Aprender a Ver. Aportes Metodológicos de la Fenomenología a una teología de los signos de los Tiempos”. In: AZCUY, Virginia Raquel; SCHICKENDANTZ, Carlos; SILVA, Eduardo (eds.). Teología de los Signos de los Tiempos Latinoamericanos. Horizontes, Criterios, y Métodos. Santiago: Ediciones Universidad Alberto Hurtado, 2013, pp. 275-315.
} 
Maradiaga, por ejemplo, dijo: "El Padre Lucio Gera es, en primer lugar un sacerdote, un hombre dedicado a la oración y al servicio a los demás... Sus palabras constituían siempre en el CELAM una visión orientadora en el discernimiento permanente de los que el Señor nos pedía a cada uno de nosotros". ${ }^{4}$ El Padre Joaquín Alliende de Chile destaca el perfil de Gera como: "un hombre de barrio, un sacerdote en el barrio, y un teólogo desde el barrio". ${ }^{45}$ En otras palabras, el teólogo que sigue el modelo de Gera tiene que acompañar al pueblo, ejercer su ministerio con y para el pueblo, y tiene que teologizar desde la perspectiva del pueblo.

Otro aporte bastante importante de Gera fue su apoyo para promover el diálogo interdisciplinario entre teología y literatura. ${ }^{46}$ En realidad, el método de Gera no es nada más que "una actitud dialogante". ${ }^{47}$ Gera cita a Dante y también un estudio de Dostoievski por Romano Guardini como ejemplos. El fruto del diálogo en términos metodológicos es el reconocimiento de la importancia del destinatario. ${ }^{48}$ Este reconocimiento es importante tanto para entrar en el mundo de literatura como en la vida de los pobres: "nos encontramos con destinatarios que no son sólo alumnos de teología y que son los pobres, o la gente de la calle, o nos encontramos con los que manejan la cultura, en el caso de ustedes con la literatura. Sobre todo nos encontramos con el poeta". ${ }^{49}$ Otra semejanza entre la literatura y la teología es la variedad de estilos. Cada poeta tiene su estilo. Por lo tanto, cada grupo nacional de teólogos tiene su propio estilo. De todas formas, el comienzo de todo diálogo teologal para Gera es la antropología teológica, "no del Dios de arriba para abajo". ${ }^{50}$

El Papa Francisco tiene un vínculo muy estrecho con Lucio Gera. El Arzobispo Jorge Mario Bergoglio quiso que Lucio Gera fuera reconocido como "Maestro en Teología" haciendo trasladar sus restos a la Cripta de la

\footnotetext{
${ }^{44}$ MARADIAGA, Oscar Rodríguez. "El sacerdote, el teólogo, y el amigo”. In: FERRARA, Ricardo; GALLI, Carlos María. (eds.) Presente y Futuro de la Teología Argentina: Homenaje a Lucio Gera. Buenos Aires: Paulinas, 1997, pp. 34-5.

${ }^{45}$ LUCO, Joaquín Alliende. "En torno a Puebla: Lucio Gera, vinculado vinculante". In: Presente y Futuro de la Teología Argentina, p. 146.

${ }^{46}$ PALUMBO, Cecilia Inés Avenatti de; CASAL, Padro Bayá; QUELAS, Juan. “"Escuchar un mundo'. Entrevista a Lucio Gera sobre el problema del método de diálogo interdisciplinario entre Teología y Literatura" Revista Teología XLVI, 9 (2009), pp. 229-47.

${ }^{47}$ Ibid., p. 238.

${ }^{48}$ Ibid., p. 232.

${ }^{49}$ Ibid.

${ }^{50}$ Ibid., p. 247.
} 
Catedral de la Iglesia Metropolitana de Buenos Aires. ${ }^{51}$ Un modelo de la teología pastoral del Papa Francisco viene de los curas que trabajan en las villas muy pobres de Buenos Aires. Bergoglio mismo creó una Vicaría distinta para los curas villeros. De hecho, Gera prologó un libro de las curas villeros para apoyar sus esfuerzos en la pastoral urbana y poner de relieve su perfil importante dentro de la iglesia local. ${ }^{52}$

Juan Carlos Scannone ha mostrado la importancia de las "perspectivas eclesiológicas de la 'Teología del Pueblo' en Argentina” y su influencia en la teología del Papa Francisco. ${ }^{53}$ Scannone subraya cuatro temas en la exhortación apostólica Evangelii Gaudium que ya eran importantes en la teología del pueblo en Argentina antes de la elección del Papa argentino. Todos los temas permiten la búsqueda de convergencias entre los opuestos, una forma de pensamiento integrante que, según Scannone, fue tomada de la teología de Gegensatz (el opuesto) en la obra de Romano Guardini del mismo nombre. ${ }^{54}$ Dice Scannone acerca de las cuatro prioridades bergoglianas:

Ya como Provincial de los jesuitas, Bergoglio enunció y, luego, como arzobispo de Buenos Aires, explicó más detalladamente, prioridades de gobierno conducentes al bien común, a saber: 1) la superioridad del todo sobre las partes (siendo más que la mera suma de las partes), 2) la de la realidad sobre la idea, 3) la de la unidad sobre el conflicto, 4) la del tiempo sobre el espacio...Más tarde -ya como Papa- Francisco introdujo las dos últimas prioridades en la encíclica a cuatro manos Lumen fidei ( $\mathrm{N}^{\text {os }} 55$ y 57). Finalmente las desarrolla y articula en Evangelii Gaudium 217-237, presentándolas como un aporte desde el pensamiento social cristiano "para la construcción de un pueblo"

\footnotetext{
${ }^{51}$ GALLI, Carlos Maria. "Lucio Gera, buen pastor y maestro en teología”. In: AZCUY, V. R.; CAAMAÑO, J. C.; GALLI, C. M. (eds.). GERA, Lucio. Meditaciones Sacerdotales. Buenos Aires: Agape, 2015, p. 11.

52 GERA, Lucio. "Prólogo". In: Equipo de Sacerdotes para las Villas de Emergencia de la Ciudad de Buenos Aires. Nuestra Mirada. Buenos Aires: Editora Patria Grande, 2009, pp. 7-16. Un versión reedita del texto con el título "Presencia de sacerdotes en las villas de Buenos Aires" se encuentra en GERA, Lucio. Meditaciones sacerdotales, pp. 177-84.

53 SCANNONE, Juan Carlos. "Papa Francisco y la teología del pueblo". Mensaje (2014), pp. 14-21. La versión original y más amplia de este ensayo se encuentra en italiano: "Papa Francesco e la teología del popolo". La Civiltà Cattolica 165 (2014), pp. 571-590.

${ }^{54}$ GUARDINI, Romano. Der Gegensatz: Versuche zu einer Philosophie des lebendig Konkreten. Mainz: Matthias-Grünewald, 1925.
} 
(en primer lugar, de los pueblos del mundo, pero también del Pueblo de Dios). ${ }^{55}$

Así vemos un hilo conductor que vincula la teología del pueblo de Lucio Gera con Evangelii Gaudium. En los dos casos el apoyo para el laico deriva de la colaboración viva entre todos los bautizados y entre todos los oficios eclesiales dentro del pueblo de Dios. De todas formas, un sentido palpable de pertenecer al pueblo es clave tanto para la teología como para la pastoral. Según el Papa Francisco, sin pertenencia no hay identidad plena en el pueblo de Dios. ${ }^{56}$

\section{La intervención del Obispo McGrath sobre los laicos durante el Concilio Vaticano II}

El tema de los pobres no fue enfatizado en los documentos conciliares del Concilio. ${ }^{57}$ Según Gustavo Gutiérrez: "[e]1 Vaticano II alude varias veces a la pobreza pero no hace de ella una de sus líneas fuerza". ${ }^{58} \mathrm{McGrath}$ participó durante el último año del Concilio en el Pacto de los Catacumbas y firmó su decreto sobre la iglesia de los pobres. Aunque la discusión de la pobreza durante los años del Concilio ha tenido sólo un impacto modesto en los textos, el tema fue elaborado a través de un libro provocante de Paul Gauthier y, también, surgió de manera clara en otros contextos y sitios. ${ }^{59}$

McGrath realizó un aporte significativo para promover, durante la segunda sesión del Concilio y antes de la reunión en la Catacumba de Domitila, la idea de una iglesia de los pobres. Marcos McGrath, en la Congregación General del Concilio del 22 de octubre de 1963, efectuó una intervención sobre el esquema de la Iglesia, capítulo tres (sobre el pueblo de

\footnotetext{
${ }^{55}$ SCANNONE, Juan Carlos. "Papa Francisco y la Teología del Pueblo”, p. 18.

${ }^{56}$ SPADARO, Antonio, S.J. "A Big Heart Open to God: The Exclusive Interview with Pope Francis”. America 20 (2013). Disponible en: <http://americamagazine.org/pope-interview>.

${ }^{57}$ Véase LOIS, Julio. Teología de la Liberación: Opción por los pobres. San José, Costa Rica: Editorial Departamento Ecuménico de Investigaciones, 1988, pp. 23-27; PELLETIER, Denis. “Une Marginalité engagée. Le Groupe 'Jésus, L'Église et les Pauvres'”, en Les Commissions Conciliares à Vatican II, pp. 63-89; y Hünermann, Peter. El Vaticano II como Software de la Iglesia Actual. Santiago de Chile: Ediciones Universidad Alberto Hurtado, 2014, pp. 293-314. ${ }^{58}$ GUTIÉRREZ, Gustavo. La Teología de la Liberación: Perspectivas. Salamanca: Ediciones Sígueme, 1972, p. 322.

${ }^{59}$ El libro de Gauthier fue Los pobres, Jesús y la Iglesia. Barcelona, 1964. La historia de las discusiones de la pobreza se encuentra en LOIS, Julio. Teología de la liberación, pp. 11-23.
} 
Dios y especialmente de los laicos). ${ }^{60}$ Comienza McGrath con su aprecio de la inclusión del tema de los laicos:

\begin{abstract}
Ahora, en el Concilio, hablamos de ellos largamente, a fin de que precisamente, lo que se debe decir acerca de ellos, encuentre, en pleno centro de la Constitución De la Iglesia, su lugar y su contenido exacto. De esto se desprende ya que el progreso respecto de los laicos es parte esencial de la renovación de la Iglesia (aggiornamento), que todos tan intensamente esperan. Ello es motivo de regocijo, creo, para todos nosotros. ${ }^{61}$
\end{abstract}

Después menciona su crítica del esquema:

¿Por qué dicen esto? La respuesta es que el capítulo falla precisamente en la segunda parte, donde trata de los laicos. En la primera parte, que trata del Pueblo de Dios en general, y que será el nuevo capítulo segundo de la Constitución, se describe admirablemente la vida sobrenatural común a todos los fieles cristianos. Todo esto debe encarnarse, por así decir, en las circunstancias concretas de cada estado en la Iglesia...La definición del laico que se propone aquí no parece apta. Se lo define, en efecto, negativamente, como aquel bautizado que ni es religioso ni pertenece al orden jerárquico; definición deficiente en cuanto procede por oposición a dos elementos que no se encuentran de ningún modo en el mismo plano... Sería entonces mejor cambiar el texto de la definición en este sentido: "El Concilio declara que los laicos son aquéllos fieles que, incorporados por el Bautismo al Pueblo de Dios, sirven a Él en el estado común de los fieles cristianos, y, por la parte que a ellos toca, ejercen la misión de la totalidad del pueblo cristiano en el mundo, pero no pertenecen al orden jerárquico. ${ }^{62}$

Lo más notable en este texto es la referencia a lo cotidiano. McGrath formula una nueva definición del laico teológicamente correcta que también

${ }^{60}$ Un poco después del intervento (el 29 de noviembre de 1963), McGrath fue elegido para una subcomisión de ocho personas que trabajaron la parte de De ecclesia sobre los laicos. Véase TURBANTI, Giovanni. "Commissione Mista per lo Schema XVIII-XIII”, p. 231.

${ }^{61}$ Archives of the University of Notre Dame, Marcos McGrath Papers (=CMCG), CMCG 5/04, "Document: Intervención sobre el cap.3 "Acerca del Pueblo de Dios y especialmente de los Laicos" de la Constitución de la Iglesia (De Ecclesia) - by McGrath in Aula 1963/1022," p. 1. Los archivos tienen los textos oficiales en latín y dos distintas traducciones en castellano. Todos fueron en la posesión de McGrath, y por eso cito las versiones en castellano como traducciones autorizadas si no textos originales.

${ }^{62}$ Ibid., pp. 1-2. 
corresponde a la realidad de las tareas del laico (o de la laica) en el mundo moderno. También insiste en que los laicos constituyen 99\% de los miembros de la Iglesia y que "la gran mayoría de los laicos no milita entre los miembros de las asociaciones apostólicas reconocidas por la Iglesia. No obstante, muchos y siempre la mayor parte de los laicos, no pertenecerán a ellas, un buen número por razones honestas: la pobreza y sus ocupaciones". ${ }^{63}$

McGrath hace además una crítica fuerte de la idea del monopolio de la cultura realizado tanto por el clero como por el clericalismo:

Sea a causa de su pobreza, sea a causa de su vocación para las tareas seculares, se dedican [laicos] enteramente a las así llamadas cosas mundanas. Hay ciertamente valores morales comprometidos en todo esto, y a la conciencia del laico pertenece ejercer su tarea con atención a los valores morales. Pero para esto sólo necesita rara vez de la intervención de la jerarquía; para obrar con prudencia le basta su conciencia formada, y la función del clero consiste más bien en formar la conciencia del laico conforme a la mente de Cristo que pronunciar sus juicios en lugar suyo. Pues, en todas las acciones y tareas puramente seculares los laicos son los que tiene autoridad incluso sobre nosotros los Obispos: como el médico, el agente policial, el jefe del Estado, a los que debemos obedecer en su propia competencia así como ellos nos obedecen a nosotros en materia religiosa. En otras palabras, ninguna razón hay para que la civilización cristiana sea eclesiástica, ni menos clerical. ${ }^{64}$

La exposición oral de McGrath comunica una teología del laicado fascinante. ${ }^{65}$ Primero, enfatiza el papel positivo del laicado tanto en la iglesia como en la sociedad. No es suficiente hablar de la tarea secular del laicado sin desarrollar lo especial de su papel y destino eclesial. Segundo, menciona que la mayor parte de los hombres viven en la pobreza y rodeados de miserias sociales y culturales. Este reconocimiento deriva de su compromiso con la Acción Católica en los EE.UU. y con los jóvenes del Colegio Saint George. Pero es aún más una proyección al futuro de CELAM y la introducción de la

\footnotetext{
${ }^{63}$ CMCG 5/03, "Document: Intervencion de Mons. Marcos McGrath CSC sobre el Esquema De Apostolatu Laicorum", p. 5.

${ }^{64}$ CMCG 5/04, "Document: Intervención sobre el cap.3 "Acerca del Pueblo de Dios y especialmente de los Laicos" de la Constitución de la Iglesia (De Ecclesia) - by McGrath in Aula 1963/1022, pp. 3-4.

${ }^{65}$ Véase el comentario en PELTON, Robert. El obispo Marcos McGrath, pp. 36-40.
} 
opción preferencial para los pobres. El Vaticano II no ha optado por los pobres tanto como harían los obispos de CELAM, pero McGrath por lo menos ha introducido el tema. Tercero, McGrath sigue su propia teología de los signos de los tiempos en su crítica del clericalismo. Es más que un mensaje moral. Es el reconocimiento de la necesidad de repensar la relación entre fe y cultura para evitar el clericalismo y presentar una visión más amplia y atractiva de la contribución de la iglesia al progreso humano.

\section{Lucio Gera y el desafío de la nueva evangelización.}

Según Lucio Gera, una de las tareas más importantes de la época postconciliar es la de dar espacio al laico. En realidad, Gera ha elaborado antes del Concilio una visión concreta para promover la colaboración con el laicado. ${ }^{66}$ Para Gera, hay que encontrar un espacio eclesial para el laicado. ${ }^{67}$ El clericalismo es entonces "como una herejía que toca la estructura misma de la Iglesia, herejía que tiende a destruir la catolicidad en su dimensión cualitativa y, por otra parte, lleva al clero, y a la iglesia que se identifica con él, al aislamiento". ${ }^{68}$ El gran pecado según Gera es considerar al estado clerical como si fuera un estado social. Como explica bien Gerardo Farrell: "dentro de la Iglesia clero y laico se distinguen y están unidos; fuera de Ella son iguales y pueden estar o no unidos en proyectos temporales." ${ }^{\prime 9}$

Gera reconoce que hemos abordado aquí una gran cuestión jurídica. ¿Qué quiere decir el estado neutral en nuestros tiempos? ¿Es la neutralidad del estado laico en realidad neutral? Estas preguntas mantienen su relevancia hoy. Gera apoya la idea de una neutralidad abierta. ${ }^{70}$ Según Gera, el estado laico se convierte en una ideología de "laicismo" precisamente cuando su apoyo a la neutralidad frente al pluralismo religioso no puede mantener una apertura a los bienes sobrenaturales de varias creencias. La declaración conciliar Dignitatis Humanae ha desarrollado un concepto del estado laical abierto a los bienes sobrenaturales y por eso puede ser un modelo. Gera dice:

\footnotetext{
${ }^{66}$ GERA, Lucio. "Reflexión sobre clero y laicado". Notas de pastoral jocista (1953), pp. 23-33.

${ }^{67}$ FARRELL, Gerardo T. "Lucio Gera y la recepción pastoral de Concilio Vaticano II en la Argentina”. Presente y Futuro de la Teología Argentina, pp. 113-4.

${ }^{68}$ Ibid.

${ }^{69}$ Ibid., p. 114.

${ }^{70}$ GERA, Lucio. “Catolicismo y el Estado Laico". In: Escritos Teológico-Pastorales de Lucio Gera, II, p. 244.
} 
La neutralidad del Estado consiste en dejar libertad a los diversos grupos religiosos y culturales, para que éstos puedan organizarse, expresarse y hacer su propuesta propia y específica, a la sociedad. El Estado no impone una determinada religión y doctrina filosófica, pero deja libres a los ciudadanos, individual o grupalmente considerados, para que éstos la propongan. ${ }^{71}$

Este espacio de libertad dentro del estado laical representa la defensa del bien común desde la teología del pueblo.

Hay más aportes que hizo Gera para promover las vocaciones de los laicos. Mencionaré solamente dos más. Primero, defendió la religiosidad popular. En 1979 McGrath fue elegido Miembro y Director del Comité Central de Coordinación de la Tercera Conferencia General de Puebla. Gera asistió como Perito Asesor en la Conferencia General y redactó el texto de Puebla 385-443 sobre la evangelización de la cultura. En este contexto Gera ha luchado para defender la religiosidad popular y su complementariedad con la teología de la liberación. ${ }^{72}$ Según Puebla, la religiosidad popular frecuentemente se inserta en la actualidad de la historia, como "un elemento dinamizador". ${ }^{73}$ Además, Gera ha jugado un gran papel en la iglesia argentina a partir de la exhortación apostólica Cristifideles Laici promulgada por San Juan Pablo II en 1988 y también en la implementación, junto con el Cardenal Pironio, de la nueva evangelización a partir de la Conferencia General de CELAM en Santo Domingo (1992).

Concretamente, la teología del pueblo de Lucio Gera incluye una reflexión muy interesante sobre el misterio de Dios en categorías familiares. ${ }^{74}$ Utiliza la espiritualidad carmelitana sobre la Paternidad de Dios y la filiación adoptiva para aplicarlas a la situación familiar de los laicos en el mundo de hoy. Dice Gera:

\footnotetext{
${ }^{71}$ Ibid., p. 245.

${ }^{72}$ Véase SCANNONE, Juan Carlos. "Los aportes de Lucio Gera a la teología en perspectiva latinoamericana”. In: Presente y Futuro de la Teología Argentina, pp. 13-141; LUCO, Joaquín Alliende. "En torno a Puebla: Lucio Gera, vinculado vinculante". In: Presente y Futuro de la Teología Argentina, p. 145.

${ }^{73}$ CELAM. Iglesia y Religosidad Popular en América Latina. Bogotá: Documentos CELAM 29, 1977, p. 293.

74 GERA, Lucio. "El Misterio de Dios expresado en categorías familiares". In: Escritos Teológico-Pastorales de Lucio Gera, II, pp. 719-36.
} 
La paternidad de Dios alcanza de algún modo a todos los hombres, porque todos ellos han sido creados por él...pero se presentan diversas situaciones. No todos responden a esta llamada mediante la fe y el amor. ${ }^{75}$

Gera estaba fascinado por la espiritualidad trinitaria de Santa Teresita del Niño Jesús. En este ensayo despliega las fundaciones bíblicas y espirituales de su acercamiento a Dios Padre. Enfatizó Gera la distancia radical entre paternidad divina y paternidad humana. La divina es el modelo de la humana y no viceversa. Ciertas personas como Santa Teresita son impulsadas por la ternura de su propio Padre y puede ser elevado a la filiación divina con la ayuda del sentido analógico de paternidad dentro de la familia. Pero Gera reconoce que no todas las familias son así. Cada familia es distinta y, por eso, cada experiencia de Dios es distinta. En fin, todo nuestro lenguaje que enfrenta el misterio inalcanzable de la Trinidad es analógico. Gera desarrolla entonces, a la luz del desafío pastoral de ayudar a la espiritualidad de la familia, una teología sapiencial de Dios muy adecuada y práctica a las circunstancias de lo cotidiano.

\section{Ecclesia crucis: La misión de los laicos dentro de la iglesia de los pobres.}

Así, tenemos dos líneas latinoamericanas en la interpretación de la teología del laicado a partir del Concilio Vaticano II. La línea de McGrath se enfoca en la tarea de leer los signos de los tiempos y así abre la historia como lugar teológico, según Schickendantz. ${ }^{76}$ Además, McGrath abre un espacio para la teología de la liberación porque firmó el Pacto de los Catacumbas y defendió enfrente de todos los obispos del Concilio la realidad de la pobreza de gran parte del pueblo de Dios. Gaudium et Spes hubiera sido mucho más concreto en este punto si habrían escuchado la voz profética de McGrath.

La línea de Gera viene de una nueva síntesis de la pastoral y la teología sistemática. Unos teólogos de la liberación han criticado la escuela argentina por haber evitado hacer un análisis crítico de la sociedad, pero en realidad no es el caso. Gera propuso la llamada teología del pueblo para escuchar al pobre en las periferias de la ciudad como el destinatario de la Palabra de Dios. Su postura incluye una teología de cultura y una apertura al diálogo entre la literatura y la teología, pero nunca abandona a los pobres. Dos características

\footnotetext{
${ }^{75}$ Ibid., p. 729.

${ }^{76}$ SCHICKENDANTZ, Carlos. "Un eclipse con dos focos”, p. 71.
} 
de la línea de Gera son la importancia de la cuestión de pertenencia en la reflexión teológica y la centralidad de la antropología teológica. Si Gaudium et Spes número 4 es el punto de partida de mucha de la reflexión teológica de McGrath, el aporte a la humanidad en comunión por autodonación de sí mismo en Gaudium et Spes número 12 es el punto de partida más característico de Gera.

¿Dónde se encuentran los hispanos en este matiz? ¿Hemos aclarado todas las dudas con que hemos comenzado? La eliminación de todas las dudas no fue el propósito de este ensayo. Pero podemos aclarar ahora el perfil latino de la iglesia con más iluminación histórica y teológica. Según Goizueta, la eclesiología de los latinos se funda en la ecclesia crucis. El evento de la cruz refleja prioridades a la vez espirituales y temporales. La mayoría del pueblo latino de Dios es pobre y por eso el discurso nuevo que favorece la liberación social mantiene su prioridad. ${ }^{77}$ Una teología latina del laicado tiene que reconocer el gran aporte de McGrath en su lectura de los signos de los tiempos. La inclusión de las personas sin documentos y sin ciudadanía tanto en la iglesia como en la sociedad sería otro signo del tiempo muy actual. La teología del pueblo introducida por Lucio Gera también es importante para la gente latina, pues la realidad de la comunión interpersonal es algo muy típico del comportamiento del latino. Además, la reflexión teológica a partir de la participación en la religiosidad popular ha sido un gran parte de la contribución de los latinos y latinas a la teología de los Estados Unidos. Por eso, ninguna de estas dos líneas puede ser rechazada ni priorizada por los latinos.

El aporte del pueblo latino sería entonces el reconocimiento de la complementariedad y convergencia de ambas líneas eclesiológicas en su propia experiencia de la iglesia. Los latinos y latinas leen los signos de los tiempos y promueven el pueblo de Dios teológicamente y en la pastoral.

\section{Apéndice: Cronología}

1924 Nacimiento de Lucio Gera (LG) en Argentina y de Marcos McGrath (MM) en la zona estadounidense de Panamá.

1947 Ordenación de LG en Buenos Aires.

1949 Ordenación de MM en la Ciudad de Panamá.

77 Véase por ejemplo TIRRES, Christopher D. “'Liberation' in the Latino(a) Context: Retrospect and Prospect”. In: VALENTÍN, Benjamín (ed.). New Horizons in Hispanic/Latino (A) Theology. Cleveland, Ohio: The Pilgrim Press, 2003, pp. 138-62. 
1954 MM fundó las Obras Sociales del Saint George en el colegio dirigido por los padres de Sagrada Cruz en Santiago de Chile. MM comienza su enseñanza en la Facultad de Teología de la PUC durante el mismo año.

1959 MM fue nombrado Decano de la Facultad de Teología de la PUCChile.

1960 MM fundó la revista Teología y Vida.

1956 LG, "Reflexión sobre clero y laicado".

1961 MM fue nombrado Obispo Auxiliar de la Ciudad de Panamá y dejó Chile.

1962 MM fue elegido miembro de la Comisión Doctrinal en el Concilio Vaticano II y trabaja como miembro del Comité Central para la Constitución Pastoral sobre la iglesia del Mundo de Hoy.

1963 El 22 de octubre de 1963 MM ofreció una exposición oral en la Congregación General LIII acerca del esquema de la Iglesia, capítulo tres (sobre el pueblo de Dios y especialmente de los laicos).

1964 MM fue nombrado primer obispo de Santiago de Veraguas.

1965 El 16 de noviembre de 1965 MM firmó el Pacto de la Catacumbas en torno a la iglesia servidora y pobre.

1966 MM, “La génesis de Gaudium et Spes,” Mensaje (1966): 495-502.

1966 LG comienza su primer periodo de tres años como Decano de la Facultad de Teología de la Universidad Católica de Argentina. También sirve como Decano en 1979-1982 y 1982-85.

1966-72: MM fue elegido Segundo Vicepresidente del CELAM.

1967 MM fue elegido Secretario General del CELAM.

1968 MM, "Un nuevo método de pastoral de la Iglesia", Criterio 1543 (1968) 134-137.

1968 MM fue Miembro del Comité Organizador y uno de los 5 ponentes de la Segunda Guerra Conferencia General del Episcopado Latinoamericano, celebrado en Medellín, Colombia. MM dio la ponencia: "Los signos de los tiempos en América Latina hoy". 
1969 MM fue nombrado Arzobispo de Panamá y sirve allí hasta 1994.

1969 LG fue nombrado miembro de la primera Comisión Teológica Internacional.

1970 LG, “Apuntes para una interpretación de la iglesia argentina”.

1971 LG, “La Aparición del fenómeno político en el campo de la reflexión teológica".

1971 El sacerdote colombiano Hector Gallegos fue secuestrado en Panamá y nunca apareció de repente. Gallegos conoció a MM en 1965 y en 1968 fue invitado por McGrath a Santiago de Veraguas para organizar la pastoral de los campesinos. Según McGrath el aporte de Gallegos fue un modelo de la nueva pastoral postconciliar.

1976 LG, "Pueblo, religión del pueblo e iglesia", Teología 27/28 (1976): 99-123.

1978 LG, "Comentarios introductorios a los capítulos de la Evangelii Nuntiandi".

1979 MM fue elegido Miembro y Director del Comité Central de Coordinación de la Tercera Conferencia General de Puebla, México. LG como Perito Asesor en la Conferencia General redactó el texto de Puebla 385-443 sobre la evangelización de la cultura.

1979 LG, "Fe y Cultura en el Documento de Puebla", Criterio 1826 (1979): 749-54.

1986 LG colaboró con Cardenal Pironio en la preparación del Sínodo sobre la vocación y la misión de los laicos.

1988 LG, "Catolicismo y Estado Laico".

1990 LG, “San Miguel: Una promesa escondida”, Voces 17 (1990): 6-20.

1991 MM creó el Departamento de Pastoral Social respondiendo así a una necesidad experimentada en la Ciudad de Panamá.

1999 LG, "El Misterio de Dios expresado en categorías familiares".

2000 Falleció MM.

2012 Falleció LG. 


\section{Referencias bibliograficas}

AZCUY, Virginia R. "La manifestación salvífica de Dios y su discernimiento en los signos de los tiempos. El giro soteriológico-pastoral en el Concilio Vaticano II". Teología y Vida 60 (2014), pp. 329-50.

AZCUY, Virginia R. "La Pobreza de la Iglesia y los signos de los tiempos. Medellín como recepción inacabada del Vaticano II". In: AZCUY, Virginia Raquel; SCHICKENDANTZ, Carlos; SILVA, Eduardo. (eds.). Teología de los Signos de los Tiempos Latinoamericanos. Horizontes, Criterios, y Métodos. Santiago de Chile: Ediciones Universidad Alberto Hurtado, 2013, pp. 89-126.

BERRÍOS, Fernando. "Antecendentes y recepción de Gaudium et Spes en Latinoamérica. Una mirada desde Chile”. In: AZCUY, Virginia Raquel; SCHICKENDANTZ, Carlos; SILVA, Eduardo. (eds.). Teología de los Signos de los Tiempos Latinoamericanos. Horizontes, Criterios, y Métodos. Santiago de Chile: Ediciones Universidad Alberto Hurtado, 2013, pp. 28-33.

CASARELLA, Peter. "Solidarity as the Fruit of Communion: Ecclesia in America, 'Post-Liberation Theology,' and the Earth". Communio: International Catholic Review 27 (2000), pp. 98-123.

CELAM. Iglesia y Religosidad Popular en América Latina. Bogotá: Documentos CELAM 29, 1977, p. 293.

ELIZONDO, Virgilio. El futuro es mestizo: vivir donde se juntan las culturas. San Antonio: Centro Cultura Méxicano Americano, 2000.

ELIZONDO, Virgilio. Jesús de Galilea: Un Dios de Sorpresas Increíbles. Chicago: Loyola University Press, 2007.

ESPÍN, Orlando. "Pentecostalism and Popular Catholicism: The Poor and Traditio". Journal of Hispanic/Latino Theology 3, no. 2 (1995), pp. 14-43.

FARRELL, Gerardo T. "Lucio Gera y la recepción pastoral de Concilio Vaticano II en la Argentina". In: FERRARA, Ricardo; GALLI, Carlos María. (eds.). Presente y Futuro de la Teología Argentina: Homenaje a Lucio Gera. Buenos Aires: Paulinas, 1997, pp. 113-4. 
GALLI, Carlos Maria. "Lucio Gera, buen pastor y maestro en teología". In: AZCUY, Virginia Raquel; CAAMAÑO, J. C.; GALLI, Carlos Maria. (eds.). Lucio Gera, Meditaciones Sacerdotales. Buenos Aires: Agape, 2015, p. 11.

GARCÍA-RIVERA,Alejandro. The Community of the Beautiful: A Theological Aesthetics. Collegeville, MN: Liturgical Press, 1999.

GARCÍA-RIVERA, Alejandro. The Garden of God: A Theological Cosmology. Minneapolis: Fortress Press, 2009.

GARCÍA-RIVERA, Alejandro. "The Church is Beautiful and Holy". In: MADGES, William; DALEY, Michael J. (eds.). The Many Marks of the Church. New London: Twenty-Third Publications, 2006, pp. 69-73.

GERA, Lucio. "Prólogo". In: Equipo de Sacerdotes para las Villas de Emergencia de la Ciudad de Buenos Aires, Nuestra Mirada Buenos Aires: Editora Patria Grande, 2009, pp. 7-16.

GERA, Lucio. "Reflexión sobre clero y laicado". Notas de pastoral jocista (1953), pp. 23-33.

GERA, Lucio. "Catolicismo y el Estado Laico". In: Escritos TeológicoPastorales de Lucio Gera, II, p. 244.

GERA, Lucio. "El Misterio de Dios expresado en categorías familiares". In: Escritos Teológico-Pastorales de Lucio Gera, II, pp. 719-36.

GOIZUETA, Roberto. Corpus Verum: Toward a Borderland Ecclesiology. Journal of Hispanic/Latino Theology (2007). Disponible en: <http:// www.latinotheology.org/node/32/print>.

GOIZUETA, Roberto. "The Symbolic Realism of U.S. Popular Catholicism”. Theological Studies 65 (2004), pp. 255-74.

GUARDINI, Romano. Der Gegensatz: Versuche zu einer Philosophie des lebendig Konkreten. Mainz: Matthias-Grünewald, 1925.

GUTIÉRREZ, Gustavo. La Teología de la Liberación: Perspectivas. Salamanca: Ediciones Sígueme, 1972.

HÜNERMANN, Peter. El Vaticano II como Software de la Iglesia Actual. Santiago de Chile: Ediciones Universidad Alberto Hurtado, 2014, pp. 293-314. 
ISASÍ-DÍAZ, Ada María. La Habana: "The City that Inhabits Me". In: TANNER, Kathryn. (eds.). Spirit in the Cities. Minneapolis: Augsburg, 2004.

LEE, Michael. "Liberation Theology's Transcendent Moment: The Work of Xavier Zubiri and Ignacio Ellacuria as Non-Contrastive Discourse". Journal of Religion 83 (2003), pp. 226-43.

LÓPEZ, José Daniel. “Aprender a Ver. Aportes Metodológicos de la Fenomenología a una teología de los signos de los Tiempos". In: AZCUY, Virginia Raquel; SCHICKENDANTZ, Carlos; SILVA, Eduardo. (eds.). Teología de los Signos de los Tiempos Latinoamericanos. Horizontes, Criterios, y Métodos. Santiago de Chile: Ediciones Universidad Alberto Hurtado, 2013, pp. 275-315.

LOIS, Julio. Teología de la Liberación: Opción por los pobres. San José, Costa Rica: Editorial Departamento Ecuménico de Investigaciones, 1988, pp. 23-27.

LUCO, Joaquín Alliende. "En torno a Puebla: Lucio Gera, vinculado vinculante". In: FERRARA, Ricardo; GALLI, Carlos María. (eds.). Presente y Futuro de la Teología Argentina: Homenaje a Lucio Gera. Buenos Aires: Paulinas, 1997, p. 146.

MARADIAGA, Oscar Rodríguez. "El sacerdote, el teólogo, y el amigo". In: FERRARA, Ricardo; GALLI, Carlos María. (eds.). Presente y Futuro de la Teología Argentina: Homenaje a Lucio Gera. Buenos Aires: Paulinas, 1997, pp. 34-5.

MATOVINA, Timothy. Latino Catholicism: Transformation in America's Largest Church. Princeton: Princeton University Press, 2012.

McGRATH, Marcos G. The Vatican Council's Teaching on the Evolution of Dogma: A Study in Nineteenth Century Theology. Rome: Pontificum Athenaeum Angelicum, 1953.

McGRATH, Marcos G. Teología y Vida 1 (1960), p. 4.

McGRATH, Marcos G. "Un nuevo método de pastoral de la Iglesia". Criterio 1543 (1968), pp. 134-37.

McGRATH, Marcos G. Los Signos de los Tiempos en América Latina Hoy: el discurso de McGrath como Vice-Presidente de CELAM durante 
la Conferencia General en Medellín. Santiago de Chile: Oficina de Catequesis, 1968.

McGRATH, Marcos G. "La Génesis de 'Gaudium et Spes"”. Mensaje 153 (1966), pp. 495-502.

McGRATH, Marcos G. CMCG 5/03, "Document: Intervencion de Mons. Marcos McGrath CSC sobre el Esquema De Apostolatu Laicorum”, p. 5.

McGRATH, Marcos G. CMCG 5/04, "Document: Intervencion sobre el cap.3 "Acerca del Pueblo de Dios y especialmente de los Laicos" de la Constitucion de la Iglesia (De Ecclesia) - Aula 1963/1022, pp. 3-4.

PALUMBO, Cecilia Inés Avenatti de; CASAL, Padro Bayá; QUELAS, Juan. "Escuchar un mundo. Entrevista a Lucio Gera sobre el problema del método de diálogo interdisciplinario entre Teología y Literatura". Revista Teología XLVI, 9 (2000), pp. 229-47.

PAREDES, Mario J. The History of the National Encuentros: Hispanic Americans in the One Catholic Church. Mahwah, N.J.: Paulist Press, 2015.

PARRA, Fredy. Desafio del tiempo, y memoria, en Signos de Estos Tiempos, pp. 47-82.

PARRA, Fredy. Esperanza en la Historia. Idea Cristiana del Tiempo. Santiago de Chile: Ediciones Universidad Alberto Hurtado, 2011, pp. 185-237.

PELLETIER, Denis. Une Marginalité engagée: Le Groupe <<Jésus, L'Église et les Pauvres>>. In: Les Commissions Conciliares à Vatican II, pp. 63-89.

PELTON, Robert S. El obispo Marcos McGrath, C.S.C. Santiago de Chile: Talleres de E.P., 1966.

RIEBE-ESTRELLA, Gary. "Pueblo and Church". In: ESPÍN, Orlando O.; DÍAZ, Miguel H. (eds.). From the Heart of Our People: Latino/a Explorations in Catholic Systematic Theology. Maryknoll, NY: Orbis Books, 1999, pp. 172-88.

SCANNONE, Juan Carlos. "Papa Francisco y la teología del pueblo". Mensaje (2014), pp. 14-21.

SCANNONE, Juan Carlos. "Papa Francesco e la teología del popolo". La Civiltà Cattolica 165 (2014), pp. 571-590. 
SCANNONE, Juan Carlos. "Los aportes de Lucio Gera a la teología en perspectiva latinoamericana". In: FERRARA, Ricardo; GALLI, Carlos María. (eds.). Presente y Futuro de la Teología Argentina: Homenaje a Lucio Gera. Buenos Aires: Paulinas, 1997, pp. 13-141.

SCHICKENDANTZ, Carlos. Una elipse con dos focos. Hacia un nuevo método teológico a partir de Gaudium et Spes. AZCUY, Virginia Raquel; SCHICKENDANTZ, Carlos; SILVA, Eduardo. (eds.). Teología de los Signos de los Tiempos Latinoamericanos. Horizontes, Criterios, y Métodos. Santiago de Chile: Ediciones Universidad Alberto Hurtado, 2013, pp. 53-87.

SPADARO, Antonio. "A Big Heart Open to God: The Exclusive Interview with Pope Francis". America 20 (2013). Disponible en: <http:// americamagazine.org/pope-interview $>$.

TAMPE, Luis A. Encuentro Nacional Hispano de Pastoral (1972-1985): An Historical and Ecclesiological Analysis, Ph.D. dissertation, The Catholic University of America, 2014.

Peter Casarella

$\mathrm{PhD}$ in Religious Studies Associate Professor of Theology at the University of Notre Dame Indiana - U.S.A E-mail: pcasarel@nd.edu

Recebido em: 15/07/14

Aprovado em: 10/12/15 